\title{
Stroke During Hospitalization
}

National Cancer Institute

\section{Source}

National Cancer Institute. Stroke During Hospitalization. NCI Thesaurus. Code C80478.

Clinical diagnosis of stroke during a hospitalization, of which stroke was not the admitting diagnosis. 